\title{
Blockade of Retinal NMDA Receptors by Sodium Nitroprusside Is Probably Due to Nitric Oxide Formation
}

\author{
Hisamitsu Ujihara ${ }^{1}$, Akinori Akaike ${ }^{2, *}$, Yutaka Tamura ${ }^{2}$, Takeharu Yokota ${ }^{2}$, \\ Masashi Sasa ${ }^{3}$, Satoshi Kashii ${ }^{4}$ and Yoshihito Honda ${ }^{4}$ \\ 'Department of Pharmacology, School of Medicine, Yamaguchi University, Ube 755, Japan \\ ${ }^{2}$ Department of Neuropharmacology, Faculty of Pharmacy and Pharmaceutical Sciences, Fukuyama University, Fukuyama 729-02, Japan \\ ${ }^{3}$ Department of Pharmacology, School of Medicine, Hiroshima University, Hiroshima 734, Japan \\ ${ }^{4}$ Department of Ophthalmology, Faculty of Medicine, Kyoto University, Kyoto 606, Japan
}

Received March 5, $1993 \quad$ Accepted March 18, 1993

\begin{abstract}
Effects of a nitric oxide (NO)-producing agent, sodium nitroprusside, on $N$-methyl-D-aspartate (NMDA) receptor activation in the cultured retinal neurons of rats were examined. NMDA in a $\mathrm{Mg}^{2+}$ free medium evoked inward currents at the resting membrane potential. Inward currents were also evoked by kainate. Sodium nitroprusside markedly reduced the NMDA-induced currents without affecting those induced by kainate. These results suggest the possible existence of a negative feed back system of NO which serves to regulate the activation of NMDA receptors in retinal neurons.
\end{abstract}

Keywords: Nitroprusside, $N$-methyl-D-aspartate (NMDA), Retina

Similar to cells in the brain, retinal neurons are highly susceptible to damage under ischemic conditions. Extensive studies on ischemia-related neuronal injury in recent years have revealed that the pathological processes in retinal ischemia may resemble those observed in the brain. Post-ischemia neuronal injury in the retina results partly from an increased release of glutamate. $N$-methyl-D-aspartate (NMDA) receptors have been postulated to play a crucial role in retinal glutamate cytotoxicity similar to that in the brain (1-3). In cultured cortical neurons, nitric oxide (NO) is considered to be a mediator of the NMDA receptor-mediated glutamate neurotoxicity $(4,5)$. It has been demonstrated that one of the NO-generating agents, sodium nitroprusside (SNP), blocked the NMDA-induced currents in the brain (6). A negative feedback system of NO that regulates the activity of NMDA receptors has since been postulated to exist in brain neurons. As NO synthesis occurs in the retina (7), NO may regulate the NMDA receptors in the retina in a manner similar to that in the brain. To verify this speculation, experiments using a conventional patch clamp technique were performed on cultured rat retinal neurons.

Primary cultures of the retinae were obtained from fetal rats (16- to 19-day gestation). Retinal tissues were

\footnotetext{
* To whom correspondence should be addressed.
}

minced, mechanically dissociated, filtered through stainless steel mesh and plated as single cell suspensions. Cultures were incubated in Eagle's minimal essential medium (Eagle's salts, Nissui, Tokyo) supplemented with 10\% heat-inactivated fetal bovine serum ( 1 - 9 days after plating) or $10 \%$ heat-inactivated horse serum (10-14 days after plating) containing $2 \mathrm{mM}$ glutamine, $11 \mathrm{mM}$ glucose, $24 \mathrm{mM}$ sodium bicarbonate and $10 \mathrm{mM}$ 4-(2-hydroxyethyl)-1-piperazine-ethane-sulphonic acid (HEPES). Retinal cultures were maintained under conditions similar to those used for cerebral neuronal cultures (5). Primary cultures of the cortical neurons were prepared and maintained as previously described (5). After a 6-day culture, growth of the non-neuronal cells was terminated by the addition of $10^{-5} \mathrm{M}$ cytosine arabinoside. Only cultures kept for 8-13 days were used for the experiments. Most of the cultured retinal cells were neuron-like in shape and were stained with anti-neurofilament protein antibody. In contrast, only a small population of cells was stained by anti-glial fibrillary acidic protein antibody.

Recordings of whole cell currents were performed by the method of Ujihara and Albuquerque (8). The bathing solution contained $165 \mathrm{mM} \mathrm{NaCl}, 5 \mathrm{mM} \mathrm{KCl}, 2 \mathrm{mM}$ $\mathrm{CaCl}_{2} 5 \mathrm{mM}$ HEPES, $10 \mathrm{mM}$ D-glucose and $0.0003 \mathrm{mM}$

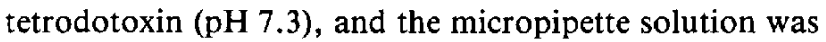
composed of $80 \mathrm{mM} \mathrm{CsCl}, 80 \mathrm{mM} \mathrm{CsF}, 10 \mathrm{mM}$ cesium 
ethylenglycol-bis-( $\beta$-aminoethylether)- $N, N, N^{\prime}, N^{\prime}$-tetraacetic acid and $10 \mathrm{mM}$ HEPES (pH 7.3). The patch microelectrodes had a resistance of $2-4 \mathrm{M} \Omega$. A fast application of NMDA or kainate was made using a U-shaped tube placed near the cell (at a distance of approximately $50 \mu \mathrm{m})$. Glycine $(10 \mu \mathrm{M})$ and strychnine $(10 \mu \mathrm{M})$ were added to the NMDA-containing solution. Whole cell currents were recorded at room temperature $\left(20-25^{\circ} \mathrm{C}\right)$ with a CEZ-2200 patch clamp system (Nihon Kohden, Tokyo).

Glycine hydrochloride, $\mathrm{K}_{4} \mathrm{Fe}(\mathrm{CN})_{6}$, kainic acid (Nacalai Tesque, Kyoto), NMDA (Sigma, St. Louis, MO, U.S.A.), SNP and strychnine nitrate (Wako, Osaka) were dissolved in the bathing solution immediately before performing the experiments.

Data are expressed as the mean \pm S.E.M. Statistical significances of the results were evaluated by Student's $t$-test.

At first, the effects of SNP on NMDA-induced currents were examined using cultured cortical neurons. SNP (300 $\mu \mathrm{M})$ inhibited NMDA-induced currents by $95.9 \pm 1.5 \%$ $(n=3)$. As Manzoni et al. (9) reported that SNP may act on NMDA receptors through its photolysis side-products, ferrocyanide ions, the effects of $\mathrm{K}_{4} \mathrm{Fe}(\mathrm{CN})_{6}$ on the NMDA-induced currents were also examined; $\mathrm{K}_{4} \mathrm{Fe}(\mathrm{CN})_{6}$ $(300 \mu \mathrm{M})$ inhibited the NMDA currents by $52.9 \pm 8.7 \%$ $(\mathrm{n}=3)$. Hemoglobin $\quad(20 \mu \mathrm{M})$ significantly $(\mathrm{P}<0.01)$ reduced the inhibitory effects of SNP, but did not affect those of $\mathrm{K}_{4} \mathrm{Fe}(\mathrm{CN})_{6} . S$-Nitrosocysteine $(300 \mu \mathrm{M})$, a NOproducing agent which does not contain ferrocyanide ions (6), also inhibited NMDA-induced currents by up to $50 \%(n=6)$, although the effects were variable probably due to its short life-time (6). Thus, we decided to examine the effects of SNP on NMDA-induced currents of the cultured retinal neurons.

Considerable inward currents were recorded from the cells with neuron-like shape when NMDA $(50 \mu \mathrm{M})$ was applied in the absence of $\mathrm{Mg}^{2+}$ at $-80 \mathrm{mV}$ (Fig. 1A-a). The decay phase of NMDA-induced currents displayed two components that were thought to represent fast and slow phases of desensitization. The NMDA-induced current was blocked by either $\mathrm{Mg}^{2+}$ (Fig. 1A-b) or $\mathrm{Zn}^{2+}$ (Fig. 1Ac). The current-voltage relationship of the response was linear when NMDA was applied in $\mathrm{Mg}^{2}$-free control medium (Fig. 1B). However, in a medium containing $\mathrm{Mg}^{2+}(100 \mu \mathrm{M})$, the current-voltage relationship displayed a characteristically negative resistant region (8, 10). $\mathrm{Zn}^{2+}(30 \mu \mathrm{M})$ also inhibited the NMDA-induced current. The inhibitory effects of $\mathrm{Zn}^{2+}$ were not dependent on the membrane potential (8). Similar results were obtained from 3 other neurons.

Since all the neurons tested responded to both the NMDA and kainate $(n=8)$, the effects of SNP $(500 \mu \mathrm{M})$ on responses evoked by these two compounds were investigated. The amplitude of kainate-induced currents did not decay during the 3-sec application period. Simultaneous application of SNP affected neither NMDA- nor kainateinduced responses in the preliminary experiments. Therefore, the effects of pretreatment with SNP were examined. SNP was applied for $3 \mathrm{~min}$ immediately before excitatory amino acid (EAA) application, and then the EAAs were applied in the absence of SNP. SNP markedly reduced the NMDA-induced currents (Fig. 2A-b). Complete recovery from the inhibitory effects of SNP was observed within $5 \mathrm{~min}$ after drug application. SNP $(500 \mu \mathrm{M})$ influenced neither kainate-induced currents (Fig. 2B-b) nor the baseline of the currents. SNP significantly $(\mathrm{P}<0.01)$ reduced the mean amplitude of the NMDA-induced currents by $77.3 \pm 7.4 \%(n=3)$, but reduced that of the kainate-induced currents only by $2.3 \pm 11.8 \%(n=3)$.

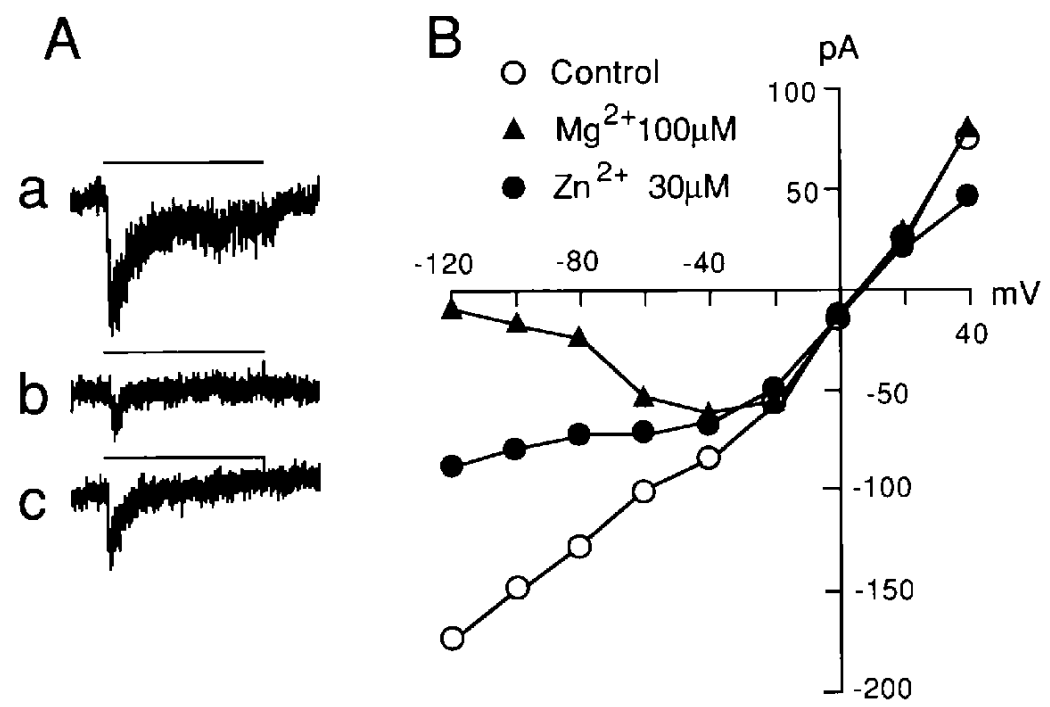

Fig. 1. NMDA-induced currents under the voltage clamp condition. NMDA was added to $\mathrm{Mg}^{2+}$-free medium in control. (A) NMDA (50 $\mu \mathrm{M})$-induced currents of the control (a), in the presence of $100 \mu \mathrm{M}$

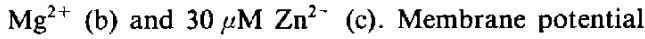
was clamped at $-80 \mathrm{mV}$. Bars over traces indicate the period of NMDA application. (B) Currentvoltage relationships of NMDA-induced currents under conditions similar to (A). The traces in (A) and data in (B) were obtained from the same cell. 
A

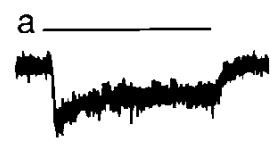

b

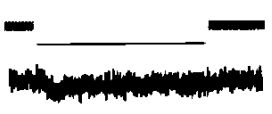

C

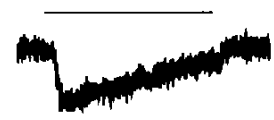

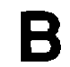

a

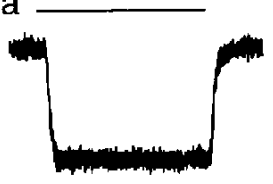

b

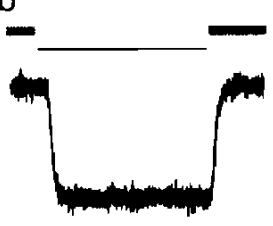

C

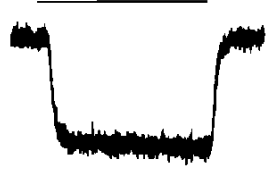

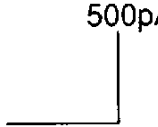

$2 \mathrm{sec}$
Fig. 2. Effects of $500 \mu \mathrm{M}$ sodium nitroprusside (SNP) on the whole cell currents induced by NMDA (A) and kainate (B). The membrane potential was clamped at $-80 \mathrm{mV}$. NMDA $(50 \mu \mathrm{M})$ was added to $\mathrm{Mg}^{2+}$-free medium. Traces were recorded under the control condition (a), immediately after 3-min SNP application (b) and on recovery ( 5 min after SNP application, c). Bar and hatched column over the traces indicate the application intervals of EAA and SNP, respectively.

The present study has demonstrated that rat retinal neurons possess both NMDA and non-NMDA receptors. The properties of the responses induced by the selective agonists of these receptors resembled those reported in brain neurons in terms of desensitization (8). Although NMDA-induced currents were suppressed by $\mathrm{Mg}^{2+}$ and $\mathrm{Zn}^{2+}$, only the former inhibition was dependent on the membrane potential because the $\mathrm{Mg}^{2+}$-induced effects were evident at potentials less than $-60 \mathrm{mV}$. These results indicate that retinal cultures possess NMDA receptors with properties similar to those of brain neurons $(8$, $10)$.

The NO-producing agent SNP is used extensively for the study of the NO effects on various organs and systems (4-6). SNP induces delayed death in cortical cultures, and SNP-induced cytotoxicity is antagonized by the NOtrapping hemoglobin $(4,5)$. Lei et al. (6) have reported that SNP inhibits NMDA-induced $\mathrm{Ca}^{2+}$ influx in cultured cortical neurons. In their study, SNP inhibits not only NMDA-induced responses but also high $\left[\mathrm{K}^{+}\right]_{0}$-induced responses. Since SNP-induced effects were relatively promiscuous, SNP may possess other effects besides its NO-producing effect. Therefore, we have examined the effects of SNP on both NMDA- and kainate-induced responses of the retinal neurons to assure the selectivity of the SNP-induced effects. In our present study, SNP inhibited NMDA-induced responses without affecting kainate-induced responses, indicating that the SNP effect in the present preparation was selective for the response mediated by the NMDA receptors of retinal neurons. This suggests that NO inhibits NMDA receptors of retinal neurons as it does in the brain (6). Our results also suggest a possible existence of a negative feedback system of NO that regulates NMDA receptor-mediated glutamate neurotoxicity in the retina. However, the SNP-induced effects may be partly due to the formation of ferrocyanide ions (9). Further studies are required to elucidate the potency of the NO-induced blockade of NMDA receptors.

\section{REFERENCES}

1 Bresnick, G.H. and Madison, W.: Excitotoxin: A possible new mechanism for the pathogenesis of ischemic retinal damage. Arch. Ophthalmol. 107, 339-341 (1989)

2 Gibson, B.L. and Reif-Lehrer, L.: $\mathrm{Mg}^{2-}$ reduces $N$-methyl-Daspartate neurotoxicity in embryonic chick neuronal retina in vitro. Neurosci. Lett. 57, 13-18 (1985)

3 Akaike, A., Tamura, Y., Sato, Y., Sasa, M., Kashii, S., Takahashi, M. and Honda, Y.: $N$-methyl-D-aspartate (NMDA) receptor-mediated glutamate cytotoxicity in primary culture of retinal neurons. Japan. J. Pharmacol. 58, Supp. I, 182P (1992)

4 Dawson, V.L., Dawson, T.M., London, E.D., Bredt, D.S. and Snyder, S.H.: Nitric oxide mediates glutamate neurotoxicity in primary cortical cultures. Proc. Natl. Acad. Sci. U.S.A. 88, $6368-6371$ (1991)

5 Tamura, Y., Sato, Y., Akaike, A. and Shiomi, H.: Mechanisms of cholecystokinin-induced protection of cultured cortical neurons against $N$-methyl-D-aspartate receptor-mediated glutamate cytotoxicity. Brain Res. 592, 317-325 (1992)

6 Lei, S.Z., Pan, Z.-H., Aggarwal, S.K., Chen, H.-S.V., Hartman, J., Sucher, N.J. and Lipton, S.A.: Effect of nitric oxide production on the redox modulatory site of the NMDA receptor-channel complex. Neuron 8, $1087-1099$ (1992)

7 Venturini, C.M., Knowles, R.G., Palmer, R.M. and Moncada, S.: Synthesis of nitric oxide in the bovine retina. Biochem. Biophys. Res. Commun. 180, 920-925 (1991)

8 Ujihara, H. and Albuquerque, E.X.: Ontogeny of $N$-methyl-Daspartate-induced current in cultured hippocampal neurons. J. Pharmacol. Exp. Ther. 263, 859-867 (1992)

9 Manzoni, O., Prezeau, L., Desagher, S. Sahuquet, A., Sladeczek, F., Bockaert, J. and Fagni, L.: Sodium nitroprusside blocks NMDA receptors via formation of ferrocyanide ions. Neuroreport 3, 77-80 (1992)

10 Nowak, L., Bregestovski, P., Ascher, P., Herbet, A. and Prochiantz, A.: Magnesium gates glutamate-activated channels in mouse central neurones. Nature 307, $462-465$ (1984) 